\title{
ON COMBINED OPERATIONAL METHOD FOR TRANSFER PROBLEMS IN HOMOGENEOUS, SPHERICAL MEDIA
}

\author{
T. H. KHO and K. K. SEN \\ Department of Mathematics, University of Singapore, Singapore
}

(Received 2 June, 1971)

\begin{abstract}
In this paper, the Combined Operational Method developed by Busbridge (1961) in connection with the radiative transfer problems in plane-parallel atmospheres has been extended to similar problems in isotropic scattering, homogeneous spherical media. The relevant auxiliary equation has been formulated, the scattering function defined and the integro-differential equation for such function deduced. For a medium having radial distribution of source in addition to the incident flux at the outer surface, the integro-differential equations for source function and emergent intensity have been established.
\end{abstract}

\section{Introduction}

Ambartzumian's physical and mathematical methods in different forms have been extensively used in solving transfer problems in plane-parallel media of finite and semiinfinite dimensions. Amongst them one can mention the method of invariant imbedding by Bellman and Kalaba $(1956,1963)$, the probabilistic method of Sobolev (1963) and Ueno (1957-1960), and the combined operational method of Busbridge (1955-1957, $1960,1961)$. Of these, the method of invariant imbedding and that based on the probability of photon emission have been extended to transfer problems in homogeneous and inhomogeneous atmospheres of spherical and cylindrical geometries. Mention can be made, in this regard, of the works of Bailey (1964), Bailey and Wing (1964), Bellman et al. (1966, 1968, 1969), Tsujita (1967), Ueno et al. (1969), Uesugi and Tsujita (1969), Leong and Sen (1968-1970), and others. All these works are based on Ambartzumian's physical method. The combined operational method, however, is based on Ambartzumian's mathematical method (cf. Kourganoff 1952).

In the present paper, the possibility of extending the combined operational method of Busbridge (1960-1961) for solving transfer problems in spherical media is examined. As a first attempt, the problem of diffuse reflection by a homogeneous, isotropically scattering, spherical medium is considered with possible radial distribution of internal sources.

\section{Model}

We consider a homogeneous isotropically scattering, spherical medium of radius $R$. The diffuse radiation field is supposed to be axially symmetric so that the source function and the intensity are azimuth-independent.

The relevant equation of transfer for the diffuse radiation field is given by

$$
\mu \frac{\partial}{\partial r} I(r, \mu)+\frac{1-\mu^{2}}{r} \frac{\partial}{\partial \mu} I(r, \mu)=-\alpha[I(r, \mu)-J(r)],
$$


where the source function $J(r)$ is given by

$$
J(r)=\frac{\bar{\omega}}{2} \int_{-1}^{1} I\left(r, \mu_{1}\right) \mathrm{d} \mu_{1}+B(r),
$$

where

$$
\begin{aligned}
& B(r)=B_{0}(r)+B_{1}(r), \\
& \bar{\omega}=\sigma / \alpha \text { and } 0 \leqslant \bar{\omega} \leqslant 1,
\end{aligned}
$$

$\bar{\omega}$ is the albedo for single scattering, $\sigma$ and $\alpha$ being scattering and attenuation coefficients respectively. The first term on the right-hand side of Equation (2.2) accounts for the diffuse radiation part of the source function. The second term $B(r)$ accounts for the reduced incident flux $B_{0}(r)$ and the radial distribution of internal sources $B_{1}(r)$, if any.

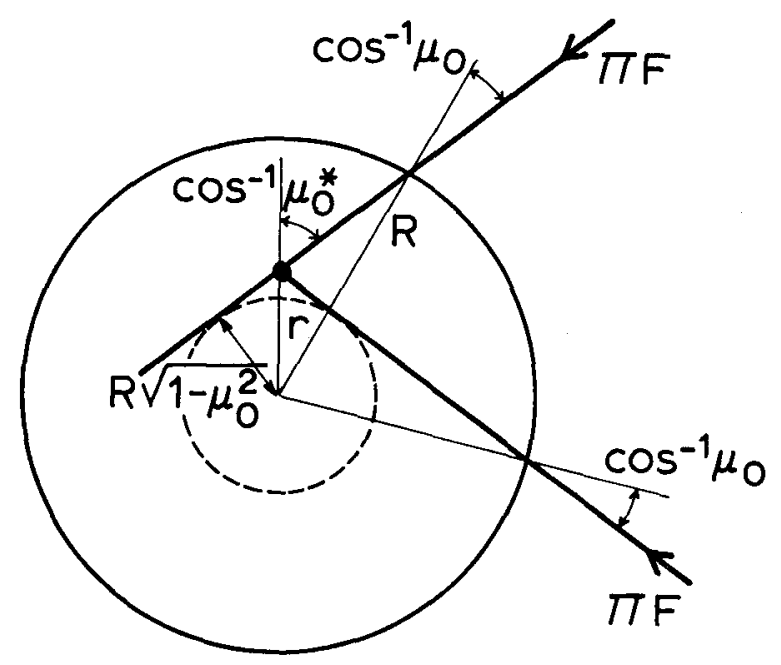

Fig. 1. Incident flux $\pi \mathrm{F}$.

Let a conical flux $\pi F$ per unit area incident at the boundary surface in a direction making an angle $\cos ^{-1} \mu_{0}$ to normal, $0<\mu_{0} \leqslant 1$, then

$$
\begin{aligned}
B_{0}(r) & =\frac{\bar{\omega} F}{4}\left(\frac{R}{r}\right)^{2} \frac{\mu_{0}}{\mu_{0}^{*}} e^{-\alpha\left(R \mu_{0}-r \mu_{0} *\right)} \cdot H\left(r-R \sqrt{1-\mu_{0}^{2}}\right) \\
& +\frac{\bar{\omega} F}{4}\left(\frac{R}{r}\right)^{2} \frac{\mu_{0}}{\mu_{0}^{*}} e^{-\alpha\left(R \mu_{0}+r \mu_{0}^{*}\right)} \cdot H\left(r-R \sqrt{1-\mu_{0}^{2}}\right),
\end{aligned}
$$

(cf. Leong and Sen (1968) for $-1 \leqslant \mu_{0}^{*} \leqslant 1$ ) where $0<\mu_{0}^{*} \leqslant 1$, and

$$
\begin{aligned}
& R^{2}\left(1-\mu_{0}^{2}\right)=r^{2}\left(1-\mu_{0}^{* 2}\right) \\
& H\left(r-R \sqrt{1-\mu_{0}^{2}}\right)=\left\{\begin{array}{lll}
1 & \text { when } & r>R \sqrt{1-\mu_{0}^{2}} \\
0 & \text { when } & r<R \sqrt{1-\mu_{0}^{2}}
\end{array}\right.
\end{aligned}
$$

is the Heaviside unit step function. 
Hence, from (2.3) and (2.5),

$$
B(r)=B_{1}(r)+\frac{\bar{\omega} F}{4}\left(\frac{R}{r}\right)^{2} \frac{\mu_{0}}{\mu_{0}^{*}} e^{-\alpha R \mu_{0}} \cdot 2 \cosh \alpha r \mu_{0}^{*} \cdot H\left(r-R \sqrt{1-\mu_{0}^{2}}\right)
$$

\section{Boundary Condition}

Let $I(r,+\mu)$ and $I(r,-\mu)$ denote respectively outward and inward intensities, $0 \leqslant \mu \leqslant 1$, then Equation (2.1) is to be solved under the boundary condition

$$
I(R,-\mu)=0
$$

that is, diffuse incident radiation at the boundary is zero.

\section{Integral Equation for the Source Function}

Following the same scheme as Cuperman et al. (1963), we obtain for the integral equation for the source function

$$
\begin{aligned}
r J(r) & =\frac{\bar{\omega}}{2} \int_{0}^{R} t J(t) K(r, t) \mathrm{d} t+r B_{1}(r)+ \\
& +\frac{\bar{\omega} F}{4} \frac{R^{2}}{r} \frac{\mu_{0}}{\mu_{0}^{*}} e^{-\alpha R \mu_{0}} \cdot 2 \cosh \alpha r \mu_{0}^{*} \cdot H\left(r-R \sqrt{1-\mu_{0}^{2}}\right),
\end{aligned}
$$

with

$$
K(r, t)=\alpha\left[E_{1}(\alpha|r-t|)-E_{1}(\alpha \mid r+t)\right]
$$

where

$$
E_{1}(x)=\int_{1}^{\infty} e^{-x u} \frac{\mathrm{d} u}{u}=\int_{x}^{\infty} e^{-u} \frac{\mathrm{d} u}{u}
$$

Since $E_{1}(x)$ is a decreasing function for $x>0$, the kernel $K(r, t)$ is non-negative. It is also symmetric, that is,

$$
K(t, r)=K(r, t)
$$

\section{Method of Solution}

\subsection{AuXiliary eQuation}

Taking $B_{1}(r)=0$, replacing $J(r)$ by $\tilde{J}(r)$ such that $J(r)=(F / 4) \bar{\omega} \tilde{J}\left(r ; R, \mu_{0}\right)$, we can write (from (2.10))

$$
\begin{aligned}
r \tilde{J}\left(r ; R, \mu_{0}\right) & =\frac{\bar{\omega}}{2} \int_{0}^{R} t \tilde{J}\left(t ; R, \mu_{0}\right) K(r, t) \mathrm{d} t+ \\
& +\frac{R^{2}}{r} \frac{\mu_{0}}{\mu_{0}^{*}} e^{-\alpha R \mu_{0}} \cdot 2 \cosh \alpha r \mu_{0}^{*} \cdot H\left(r-R \sqrt{1-\mu_{0}^{2}}\right)
\end{aligned}
$$

This is the auxiliary equation. 
The solution of Equation (3.1.1) can be expressed as a Neumann series provided it is convergent. We rewrite Equation (3.1.1) as

$$
\begin{aligned}
r \tilde{J}\left(r ; R, \mu_{0}\right) & ={ }_{R, \mu_{0}} L_{r}\left\{t \tilde{J}\left(t ; R, \mu_{0}\right)\right\}+ \\
& +\frac{R^{2}}{r} \frac{\mu_{0}}{\mu_{0}^{*}} e^{-\alpha R \mu_{0}} \cdot 2 \cosh \alpha r \mu_{0}^{*} \cdot H\left(r-R \sqrt{1-\mu_{0}^{2}}\right),
\end{aligned}
$$

where the linear operator $R, \mu_{0} L_{r}$ is defined between $0 \leqslant t \leqslant R$ and is given by

$$
{ }_{R, \mu_{0}} L_{r}\left\{f\left(t ; R, \mu_{0}\right)\right\}=\frac{\bar{\omega}}{2} \int_{0}^{R} f\left(t ; R, \mu_{0}\right) K(r, t) \mathrm{d} t \quad(0 \leqslant \bar{\omega} \leqslant 1) .
$$

Defining $r \tilde{J}\left(r ; R, \mu_{0}\right)$ as the $N$-solution of Equation (3.1.1), we have

$$
r \tilde{J}\left(r ; R, \mu_{0}\right)=\sum_{\gamma=0}^{\infty} R, \mu_{0} L_{r}^{\gamma}\left\{g\left(t ; R, \mu_{0}\right)\right\} \quad(\gamma=0,1,2, \ldots)
$$

where

$$
g\left(r ; R, \mu_{0}\right)=\frac{R^{2}}{r} \frac{\mu_{0}}{\mu_{0}^{*}} e^{-\alpha R \mu_{0}} \cdot 2 \cosh \alpha r \mu_{0}^{*} \cdot H\left(r-R \sqrt{1-\mu_{0}}\right) .
$$

${ }_{R, \mu_{0}} L_{r}^{\gamma}$ is the $\gamma$ th iterate of the operator ${ }_{R, \mu_{0}} L_{r}$ and

$$
R, \mu_{0} L_{r}^{0}\left\{g\left(t ; R, \mu_{0}\right)\right\}=g\left(r ; R, \mu_{0}\right) .
$$

\subsection{EXISTENCE AND UNIQUENESS OF THE $N$-SOLUTION OF THE AUXILIARY EQUATION}

The convergence of series (3.1.4) has been proved by Cuperman et al. (1963) and Germegenova (1966). However, we shall follow a scheme for its proof, which closely resembles that of Busbridge (1961).

THEOREM: Let $g\left(t ; R, \mu_{0}\right)$ be a function such that

$$
\left|g\left(t ; R, \mu_{0}\right)\right| \leqslant K \quad \text { (finite non-negative constant) }
$$

for $0 \leqslant t \leqslant R$, then

where

$$
\left|{ }_{R, \mu_{0}} \mathrm{~L}_{r}^{\gamma}\left\{g\left(t ; R, \mu_{0}\right)\right\}\right| \leqslant K \chi^{\gamma},
$$

$$
\chi=\left.\right|_{R, \mu_{0}} \mathbf{L}_{r}\{1\} \mid .
$$

Therefore, the series

$$
\sum_{\gamma=0}^{\infty} R, \mu_{0} L_{r}^{\gamma}\left\{g\left(t ; R, \mu_{0}\right)\right\}
$$

is uniformly convergent, if

$$
0 \leqslant \chi<1 \text {. }
$$

From (3.1.3) and (3.2.1)

$$
\left.\right|_{R, \mu_{0}} L_{r}\left\{g\left(t ; R, \mu_{0}\right)\right\} \mid \leqslant K \chi,
$$


where

$$
\chi=\left.\right|_{R, \mu_{0}} L_{r}\{1\} \mid \text {. }
$$

Since $K(r, t)$ is non-negative

$$
\begin{aligned}
0 \leqslant \chi & =\frac{\bar{\omega}}{2} \int_{0}^{R} K(r, t) \mathrm{d} t \\
& \leqslant \frac{\bar{\omega}}{2} \int_{0}^{\infty} K(r, t) \mathrm{d} t .
\end{aligned}
$$

Writing $\int_{0}^{\infty} K(r, t) \mathrm{d} t$ in the explicit form by (2.11) and (2.12), and interchanging the order of integration, we have

$$
\int_{0}^{\infty} K(r, t) \mathrm{d} t=2\left[1-E_{2}(\alpha r)\right]
$$

Therefore, (3.2.7) gives

$$
0 \leqslant \chi \leqslant \bar{\omega}\left[1-E_{2}(\alpha r)\right] \leqslant 1-E_{2}(\alpha r) \quad(0 \leqslant \bar{\omega} \leqslant 1) .
$$

As

$$
\frac{e^{-x}}{x+n}<E_{n}(x) \leqslant \frac{e^{-x}}{x+n-1} \quad(x \geqslant 0, \quad n \geqslant 0)
$$

(cf. Hopf (1934), p. 26, Equation (75)). In particular,

$$
0 \leqslant \frac{e^{-x}}{x+2}<E_{2}(x) \leqslant \frac{e^{-x}}{x+1} \leqslant 1
$$

that is,

$$
0<E_{2}(x) \leqslant 1 \text {. }
$$

Hence, from (3.2.9),

$$
0 \leqslant \chi<1 \text {. }
$$

By iteration, the result (3.2.6) can be generalised to (3.2.2). Then it follows from (3.2.2) that

$$
\left|\sum_{\gamma=0}^{\infty} R, \mu_{0} L_{r}^{\gamma}\left\{g\left(t ; R, \mu_{0}\right)\right\}\right| \leqslant \sum_{\gamma=0}^{\infty}\left|R, \mu_{0} L_{r}^{\gamma}\left\{g\left(t ; R, \mu_{0}\right)\right\}\right|=K \sum_{\gamma=0}^{\infty} \chi^{\gamma}=\frac{K}{1-\chi} .
$$

This proves that the series (3.2.4) is uniformly convergent. Therefore the $N$-solution of the auxiliary equation exists.

Now if

$$
\Phi\left(r ; R, \mu_{0}\right)=\frac{\bar{\omega}}{2} \int_{0}^{R} \Phi\left(t ; R, \mu_{0}\right) K(r, t) \mathrm{d} t
$$


is a homogeneous integral equation corresponding to the non-homogeneous auxiliary Equation (3.1.1), in order that the non-homogeneous equation has one and only one solution, the homogeneous Fredholm integral equation (3.2.14) must have the trivial solution (cf. Tricomi (1957), p. 64).

\subsection{EMERGENT INTENSITY}

The outward and inward intensities of the internal diffuse radiation field are given by

$$
I\left(r,+\mu^{*}\right)=\int_{R \sqrt{1-\mu^{2}}}^{r} J(t) e^{-\alpha\left(r \mu^{*}-t \mu^{\prime}\right)} \frac{\alpha \mathrm{d} t}{\mu^{\prime}}+\int_{R \sqrt{1-\mu^{2}}}^{R} J(t) e^{-\alpha\left(r \mu^{*}+t \mu^{\prime}\right)} \frac{\alpha \mathrm{d} t}{\mu^{\prime}}
$$

and

$$
I\left(r,-\mu^{*}\right)=\int_{r}^{R} J(t) e^{\alpha\left(r \mu^{*}-t \mu^{\prime}\right)} \frac{\alpha \mathrm{d} t}{\mu^{\prime}}
$$

where

$$
0 \leqslant \mu^{*}, \quad \mu^{\prime} \leqslant 1
$$

and

$$
r^{2}\left(1-\mu^{* 2}\right)=t^{2}\left(1-\mu^{\prime 2}\right)=R^{2}\left(1-\mu^{2}\right),
$$

(cf. Cuperman et al. (1963), Equation (3.4)).

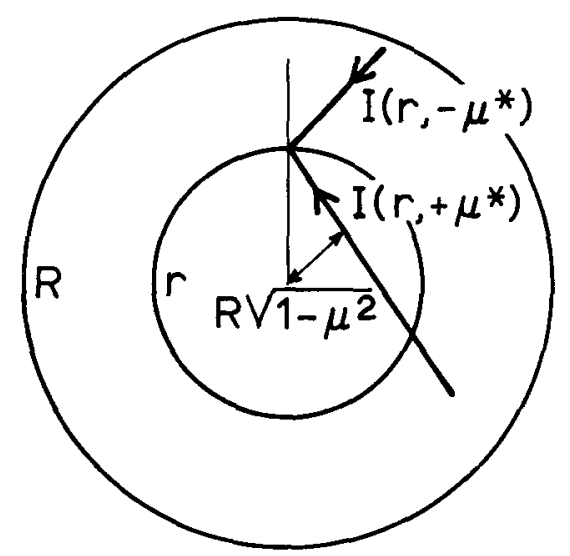

Fig. 2. The inward intensity $I\left(r,-\mu^{*}\right)$ and the outward intensity $I\left(r,+\mu^{*}\right)$.

Hence, the expression for the emergent intensity assumes the form

$$
I(R, \mu)=\int_{R \sqrt{1-\mu^{2}}}^{R} J(r) e^{-\alpha\left(R \mu-r \mu^{*}\right)} \frac{\alpha \mathrm{d} r}{\mu^{*}}+\int_{R \sqrt{1-\mu^{2}}}^{R} J(r) e^{-\alpha\left(R \mu+r \mu^{*}\right)} \frac{\alpha \mathrm{d} r}{\mu^{*}},
$$


or from $(2.7)$

$$
I(R, \mu)=\int_{0}^{R} J(r) e^{-\alpha R \mu} \cdot 2 \cosh \alpha r \mu^{*} \cdot \frac{\alpha \mathrm{d} r}{\mu^{*}} \cdot H\left(r-R \sqrt{1-\mu^{2}}\right),
$$

where $J(r)$ is given by $(2.10), 0<\mu, \mu^{*} \leqslant 1$, and

$$
r^{2}\left(1-\mu^{* 2}\right)=R^{2}\left(1-\mu^{2}\right) \text {. }
$$

In particular, when $B_{1}(r)=0,(3.3 .5)$ becomes

$$
I(R, \mu)=\frac{\sigma F}{4} \int_{0}^{R} \tilde{J}\left(r ; R, \mu_{0}\right) e^{-\alpha R \mu} \cdot 2 \cosh \alpha r \mu^{*} \cdot \frac{\alpha d r}{\mu^{*}} \cdot H\left(r-R \sqrt{1-\mu^{2}}\right),
$$

where $\tilde{J}\left(r ; R, \mu_{0}\right)$ is given by (3.1.1), $0<\mu, \mu^{*}, \mu_{0} \leqslant 1$ and

$$
r^{2}\left(1-\mu^{* 2}\right)=R^{2}\left(1-\mu^{2}\right) \text {. }
$$

\subsection{SCATTERING FUNCTION AND ITS RECIPROCITY}

From (3.3.7)

$$
\begin{aligned}
I(R, \mu)= & \frac{\sigma F}{4} \int_{0}^{R} \tilde{J}\left(r ; R, \mu_{0}\right) e^{-\alpha R \mu} \times \\
& \times 2 \cosh \alpha r \mu_{*} \cdot\left(\frac{\mu}{\mu^{*}}\right) \frac{\mathrm{d} r}{\mu} \cdot H\left(r-R \sqrt{1-\mu^{2}}\right)= \\
= & \frac{\sigma F}{4 \mu} \int_{0}^{R} \tilde{J}\left(r ; R, \mu_{0}\right) e^{-\alpha R \mu} \times \\
& \times 2 \cosh \alpha r \mu^{*} \cdot\left(\frac{\mu}{\mu^{*}}\right) \mathrm{d} r \cdot H\left(r-R \sqrt{1-\mu^{2}}\right) .
\end{aligned}
$$

Therefore we define

$$
\begin{aligned}
S\left(\mu, \mu_{0}, R\right)= & \sigma \int_{0}^{R} \tilde{J}\left(r ; R, \mu_{0}\right) e^{-\alpha R \mu} \cdot 2 \cosh \alpha r \mu^{*} \cdot\left(\frac{\mu}{\mu^{*}}\right) \mathrm{d} r \times \\
& \times H\left(r-R \sqrt{1-\mu^{2}}\right),
\end{aligned}
$$

where $J\left(r ; R, \mu_{0}\right)$ is given by (3.1.1),

$$
0<\mu_{0}, \mu, \mu^{*} \leqslant 1 \text {, and } R^{2}\left(1-\mu^{2}\right)=r^{2}\left(1-\mu^{* 2}\right) \text {. }
$$

Hence the intensity $I(R, \mu)$ for $B_{1}(r)=0$ is given by

$$
I(R, \mu)=\frac{F}{4 \mu} S\left(\mu, \mu_{0}, R\right)
$$

$S\left(\mu, \mu_{0}, R\right)$ is the scattering function. 


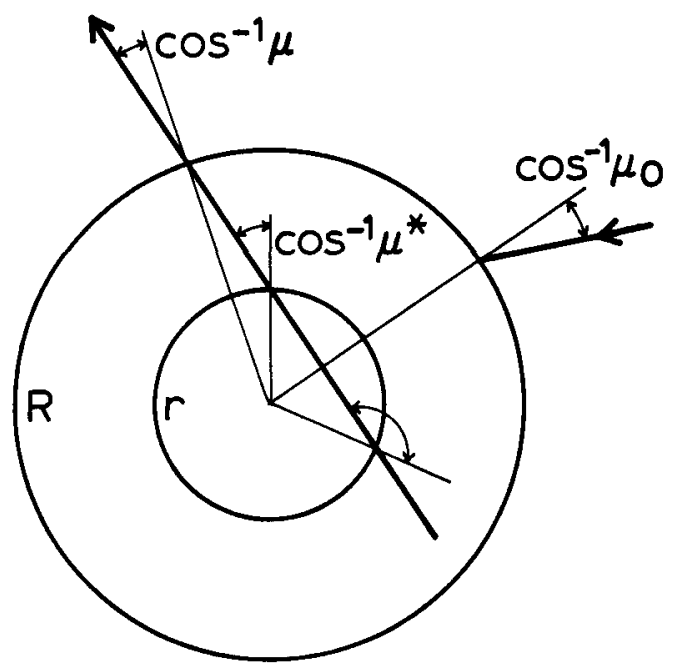

Fig. 3. The scattering function $S\left(\mu, \mu_{0}, R\right)$, connecting incident and emergent intensities.

We can show that

$$
S\left(\mu, \mu_{0}, R\right)=S\left(\mu_{0}, \mu, R\right) .
$$

From (3.1.4) and (3.1.5)

$$
\begin{aligned}
& S\left(\mu, \mu_{0}, R\right)=\sum_{\gamma=0}^{\infty} \sigma \int_{0}^{R}\left(\frac{R}{r}\right) \frac{\mu}{\mu^{*}} e^{-\alpha R \mu} \cdot 2 \cosh \alpha r \mu^{*} \cdot H\left(r-R \sqrt{1-\mu^{2}}\right) \mathrm{d} r \times \\
& \quad{ }_{R, \mu_{0}} \mathrm{~L}_{r}^{\gamma}\left\{\left(\frac{R}{t}\right) \frac{\mu_{0}}{\mu_{0}^{\prime}} e^{-\alpha R \mu_{0}} \cdot 2 \cosh \alpha t \mu_{0}^{\prime} \cdot H\left(t-R \sqrt{1-\mu_{0}^{2}}\right)\right\}
\end{aligned}
$$

Now if $f(t)$ and $g(r)$ are two non-negative functions for $0 \leqslant t, r \leqslant R$, then from (3.1.3) and (2.13)

$$
\begin{aligned}
\int_{0}^{R} g(r ; R, \mu)_{R, \mu_{0}} \mathrm{~L}_{r} & \left\{f\left(t ; R, \mu_{0}\right)\right\} \mathrm{d} r= \\
& =\frac{\bar{\omega}}{2} \int_{0}^{R} g(r ; R, \mu) \mathrm{d} r \int_{0}^{R} f\left(t ; R, \mu_{0}\right) K(r, t) \mathrm{d} t= \\
& =\frac{\bar{\omega}}{2} \int_{0}^{R} f\left(t ; R, \mu_{0}\right) \mathrm{d} t \int_{0}^{R} g(r ; R, \mu) K(r, t) \mathrm{d} r= \\
& =\int_{0}^{R} f(t ; R, \mu)_{R, \mu_{0}} L_{t}\{g(r ; R, \mu)\} \mathrm{d} t .
\end{aligned}
$$


Hence,

$$
\int_{0}^{R} g(r ; R, \mu)_{R, \mu_{0}} \mathrm{~L}_{r}\left\{f\left(t ; R, \mu_{0}\right)\right\} \mathrm{d} r=\int_{0}^{R} f\left(t ; R, \mu_{0}\right)_{R, \mu} \mathrm{L}_{t}\{g(r ; R, \mu)\} \mathrm{d} t .
$$

By induction, the result can be generalised to $\gamma$ th iterate as

$$
\int_{0}^{R} g(r ; R, \mu)_{R, \mu_{0}} \mathrm{~L}_{r}^{\gamma}\left\{f\left(t ; R, \mu_{0}\right)\right\} \mathrm{d} r=\int_{0}^{R} f\left(t ; R, \mu_{0}\right)_{R, \mu} \mathrm{L}_{t}^{\gamma}\{g(r ; R, \mu)\} \mathrm{d} t .
$$

Hence from (3.4.5)

$$
\begin{aligned}
& S\left(\mu, \mu_{0}, R\right)=\sum_{\gamma=0}^{\infty} \sigma \int_{0}^{R}\left(\frac{R}{t}\right) \frac{\mu_{0}}{\mu_{0}^{\prime}} e^{-\alpha R \mu_{0}} \cdot 2 \cosh \alpha t \mu_{0}^{\prime} \cdot H\left(t-R \sqrt{1-\mu_{0}^{2}}\right) \mathrm{d} t \times \\
& \quad \times R_{R, \mu} L_{t}^{\gamma}\left\{\left(\frac{R}{r}\right) \frac{\mu}{\mu^{*}} e^{-\alpha R \mu} \cdot 2 \cosh \alpha r \mu^{*} \cdot H\left(r-R \sqrt{1-\mu^{2}}\right)\right\}=S\left(\mu_{0}, \mu, R\right) .
\end{aligned}
$$

The inversion of the order of integration and summation in (3.4.9) is justified since the functions $f(t)$ and $g(r)$ are non-negative.

\subsection{THE INTEGRO-DIFFERENTIAL EQUATION FOR THE SCATTERING FUNCTION}

First we derive a homogeneous integral equation corresponding to the auxiliary Equation (3.1.1).

Since

we have

$$
R^{2}\left(1-\mu_{0}^{2}\right)=r^{2}\left(1-\mu_{0}^{* 2}\right)
$$

$$
\frac{\partial \mu_{0}^{*}}{\partial R}=\frac{-R\left(1-\mu_{0}^{2}\right)}{r^{2} \mu_{0}^{*}}
$$

and

$$
\frac{\partial \mu_{0}^{*}}{\partial \mu_{0}}=\left(\frac{R}{r}\right)^{2} \frac{\mu_{0}}{\mu_{0}^{*}}
$$

Differentiating Equation (3.1.1) with respect to $R$ and using (3.5.1) and remembering [cf. (A.1) of Appendix A] that

$$
K(r, R)=\frac{\alpha R}{r} \int_{0}^{1} e^{-\alpha R \mu_{1}} \cdot 2 \cosh \alpha r \mu_{1}^{*} \cdot \frac{\mathrm{d} \mu_{1}}{\mu_{1}^{*}} \cdot H\left(\mu_{1}-\sqrt{1-\frac{r^{2}}{R^{2}}}\right),
$$

we obtain

$$
\begin{aligned}
& \frac{\partial}{\partial R}\left[r J\left(r ; R, \mu_{0}\right)\right]=\frac{\bar{\omega}}{2} \int_{0}^{R} \frac{\partial}{\partial R}\left[t \tilde{J}\left(t ; R, \mu_{0}\right)\right] K(r, t) \mathrm{d} t-Q\left(R, r, \mu_{0}\right)+ \\
& +\frac{\sigma}{2} \frac{R^{2}}{r} \tilde{J}\left(R ; R, \mu_{0}\right) \int_{0}^{1} e^{-\alpha R \mu_{1}} \cdot 2 \cosh \alpha r \mu_{1}^{*} \cdot \frac{\mathrm{d} \mu_{1}}{\mu_{1}^{*}} \cdot H\left(\mu_{1}-\sqrt{1-\frac{r^{2}}{R^{2}}}\right)+
\end{aligned}
$$




$$
\begin{aligned}
& +\left[\frac{2}{R}+\frac{R\left(1-\mu_{0}^{2}\right)}{r^{2} \mu_{0}^{* 2}}-\alpha \mu_{0}\right] \cdot \frac{R^{2}}{r} \frac{\mu_{0}}{\mu_{0}^{*}} e^{-\alpha R \mu_{0}} \cdot 2 \cosh \alpha r \mu_{0}^{*} \times \\
& \times H\left(r-R \sqrt{1-\mu_{0}^{2}}\right)+ \\
& +\left[-\frac{\alpha R\left(1-\mu_{0}^{2}\right)}{r \mu_{0}^{*}}\right] \cdot \frac{R^{2}}{r} \frac{\mu_{0}}{\mu_{0}^{*}} e^{-\alpha R \mu_{0}} \cdot 2 \sinh \alpha r \mu_{0}^{*} \cdot H\left(r-R \sqrt{1-\mu_{0}^{2}}\right)
\end{aligned}
$$

where

$$
\begin{aligned}
& Q\left(R, r, \mu_{0}\right)=2 \frac{R^{2}}{\gamma} \frac{\mu_{0}}{\mu_{0}^{*}} \sqrt{1-\mu_{0}^{2}} \delta\left(r-R \sqrt{1-\mu_{0}^{2}}\right) \times \\
& \times e^{-\alpha R \mu_{0}} \cosh \alpha r \mu_{0}^{*}
\end{aligned}
$$

as $(\mathrm{d} H / \mathrm{d} x)=\delta(x)$, a $\delta$-function.

Now differentiating Equation (3.1.1) with respect to $\mu_{0}$, using (3.5.2) and multiplying it by $\left(1-\mu_{0}^{2}\right) / R \mu_{0}$, we obtain

$$
\begin{aligned}
\frac{1-\mu_{0}^{2}}{R \mu_{0}} \frac{\partial}{\partial \mu_{0}}[ & {\left[r \tilde{J}\left(r ; R, \mu_{0}\right)\right]=\frac{\bar{\omega}}{2} \int_{0}^{R} \frac{1-\mu_{0}^{2}}{R \mu_{0}} \frac{\partial}{\partial \mu_{0}}\left[t \tilde{J}\left(t ; R, \mu_{0}\right)\right] K(r, t) \mathrm{d} t+Q\left(R, r, \mu_{0}\right)+} \\
& +\left[\frac{1-\mu_{0}^{2}}{R \mu_{0}^{2}}-\frac{R\left(1-\mu_{0}^{2}\right)}{r^{2} \mu_{0}^{* 2}}-\frac{\alpha\left(1-\mu_{0}^{2}\right)}{\mu_{0}}\right] \cdot \frac{R^{2}}{r} \frac{\mu_{0}}{\mu_{0}^{*}} e^{-\alpha R \mu_{0}} \cdot 2 \cosh \alpha r \mu_{0}^{*} \times \\
& \times H\left(r-R \sqrt{1-\mu_{0}^{2}}\right)+ \\
+ & {\left[\frac{\alpha R\left(1-\mu_{0}^{2}\right)}{r \mu_{0}^{*}}\right] \cdot \frac{R^{2}}{r} \frac{\mu_{0}}{\mu_{0}^{*}} e^{-\alpha R \mu_{0}} \cdot 2 \sinh \alpha r \mu_{0}^{*} \cdot H\left(r-R \sqrt{1-\mu_{0}^{2}}\right) . }
\end{aligned}
$$

From Equation (3.1.1), replacing $\mu_{0}$ by $\mu_{1}$, we obtain

$$
\begin{aligned}
\int_{0}^{1} r \tilde{J}\left(r ; R, \mu_{1}\right) & \frac{\mathrm{d} \mu_{1}}{\mu_{1}}=\frac{\bar{\omega}}{2} \int_{0}^{R} K(r, t) \mathrm{d} t \int_{0}^{1} t \tilde{J}\left(t ; R, \mu_{1}\right) \frac{\mathrm{d} \mu_{1}}{\mu_{1}}+ \\
+ & \frac{R^{2}}{r} \int_{0}^{1} e^{-\alpha R \mu_{1}} \cdot 2 \cosh \alpha r \mu_{1}^{*} \cdot \frac{\mathrm{d} \mu_{1}}{\mu_{1}^{*}} \cdot H\left(\mu_{1}-\sqrt{1-\frac{r^{2}}{R^{2}}}\right)
\end{aligned}
$$

Interchange of the order of integration is permitted in (3.5.5) as the integrands are non-negative. Further, the validity of the steps (3.5.4), (3.5.5) and (3.5.6) can be justified from the theory of $\mathrm{N}$-solutions (cf. Appendix B). Furthermore, from Equation (3.1.1), we obtain

$$
\begin{aligned}
& -\frac{1+\mu_{0}^{2}}{R \mu_{0}^{2}}\left[r \tilde{J}\left(r ; R, \mu_{0}\right)\right]+\frac{\alpha}{\mu_{0}}\left[r \tilde{J}\left(r ; R, \mu_{0}\right)\right]= \\
& =\frac{\bar{\omega}}{2} \int_{0}^{R}\left\{-\frac{1+\mu_{0}^{2}}{R \mu_{0}^{2}}\left[t \tilde{J}\left(t ; R, \mu_{0}\right)\right]+\frac{\alpha}{\mu_{0}}\left[t \tilde{J}\left(t ; R, \mu_{0}\right)\right]\right\} \cdot K(r, t) \mathrm{d} t+ \\
& +\left[-\frac{1+\mu_{0}^{2}}{R \mu_{0}^{2}}+\frac{\alpha}{\mu_{0}}\right] \cdot \frac{R^{2}}{r} \frac{\mu_{0}}{\mu_{0}^{*}} e^{-\alpha R \mu_{0}} \cdot 2 \cosh \alpha r \mu_{0}^{*} \cdot H\left(r-R \sqrt{1-\mu_{0}^{2}}\right) .
\end{aligned}
$$


Equations (3.5.4), (3.5.5), (3.5.6) and (3.5.7) will then lead to the following homogeneous integral equation with the same kernel as the auxiliary equation

$$
\Phi_{1}\left(r ; R, \mu_{0}\right)=\frac{\bar{\omega}}{2} \int_{0}^{R} \Phi_{1}\left(r ; R, \mu_{0}\right) K(r, t) \mathrm{d} t,
$$

where

$$
\begin{aligned}
\Phi_{1}\left(r ; R, \mu_{0}\right) & =\frac{\partial}{\partial R}\left[r \tilde{J}\left(r ; R, \mu_{0}\right)\right]+\frac{1-\mu_{0}^{2}}{R \mu_{0}} \frac{\partial}{\partial \mu_{0}}\left[r \tilde{J}\left(r ; R, \mu_{0}\right)\right]- \\
& -\frac{1+\mu_{0}^{2}}{R \mu_{0}^{2}}\left[r \tilde{J}\left(r ; R, \mu_{0}\right)\right]+\frac{\alpha}{\mu_{0}}\left[r \tilde{J}\left(r ; R, \mu_{0}\right)\right]- \\
& -\frac{\sigma}{2} \tilde{J}\left(R ; R, \mu_{0}\right) \int_{0}^{1} r \tilde{J}\left(r ; R, \mu_{1}\right) \frac{\mathrm{d} \mu_{1}}{\mu_{1}} .
\end{aligned}
$$

This may be considered as the homogeneous integral equation corresponding to the non-homogeneous auxiliary equation and for the auxiliary equation to have a unique solution

Therefore

$$
\Phi_{1}\left(r ; R, \mu_{0}\right)=0 \text {. }
$$

$$
\begin{aligned}
\frac{\partial}{\partial R}\left[r \tilde{J}\left(r ; R, \mu_{0}\right)\right]+\frac{1-\mu_{0}^{2}}{R \mu_{0}} \frac{\partial}{\partial \mu_{0}}\left[r \tilde{J}\left(r ; R, \mu_{0}\right)\right]- \\
-\frac{1+\mu_{0}^{2}}{R \mu_{0}^{2}}\left[r \tilde{J}\left(r ; R, \mu_{0}\right)\right]+\frac{\alpha}{\mu_{0}}\left[r J\left(r ; R, \mu_{0}\right)\right]= \\
=\frac{\sigma}{2} \tilde{J}\left(R ; R, \mu_{0}\right) \int_{0}^{1} r \tilde{J}\left(r ; R, \mu_{1}\right) \frac{\mathrm{d} \mu_{1}}{\mu_{1}} .
\end{aligned}
$$

Now, multiplying Equation (3.5.11) by

$$
\sigma \frac{\mu}{\mu^{*}} e^{-\alpha R \mu} \cdot 2 \cosh \alpha r \mu^{*} \cdot \mathrm{d} r \cdot H\left(r-R \sqrt{1-\mu^{2}}\right),
$$

integrating it over $(0, R)$, and inverting the order of integration on the right-hand side (which is permitted because the integrand is non-negative), we get

$$
\begin{aligned}
\sigma \int_{0}^{R} \frac{\mu}{\mu^{*}} e^{-\alpha R \mu} \cdot 2 \cosh \alpha r \mu^{*} \cdot\left[\frac{\partial}{\partial R} f\left(r ; R, \mu_{0}\right)\right] \mathrm{d} r \cdot H\left(r-R \sqrt{1-\mu^{2}}\right)+ \\
+\frac{1-\mu_{0}^{2}}{R \mu_{0}} \frac{\partial}{\partial \mu_{0}} S\left(\mu, \mu_{0}, R\right)-\frac{1+\mu_{0}^{2}}{R \mu_{0}^{2}} S\left(\mu, \mu_{0}, R\right)+\frac{\alpha}{\mu_{0}} S\left(\mu, \mu_{0}, R\right)= \\
=\frac{\sigma}{2} \tilde{J}(R ; R, \mu) \int_{0}^{1} S\left(\mu, \mu_{1}, R\right) \frac{\mathrm{d} \mu_{1}}{\mu_{1}}
\end{aligned}
$$


where $S\left(\mu, \mu_{0}, R\right)$ is given by (3.4.2). Again, since $R^{2}\left(1-\mu^{2}\right)=r^{2}\left(1-\mu^{* 2}\right)$ we have

$$
\frac{\partial \mu^{*}}{\partial R}=\frac{-R\left(1-\mu^{2}\right)}{r^{2} \mu^{*}}
$$

and

$$
\frac{\partial \mu^{*}}{\partial \mu}=\left(\frac{R}{r}\right)^{2} \frac{\mu}{\mu^{*}} .
$$

Differentiating (3.4.2) with respect to $R$ and using (3.5.13), we obtain

$$
\begin{aligned}
& \frac{\partial}{\partial R} S\left(\mu, \mu_{0}, R\right)=\sigma \tilde{J}\left(R ; R, \mu_{0}\right)\left[1+e^{-2 \alpha R \mu}\right]- \\
& -\sigma \sqrt{1-\mu^{2}} \cdot \tilde{J}\left(R \sqrt{1-\mu^{2}} ; R, \mu_{0}\right) \cdot \frac{\mu}{\mu^{*}} e^{-\alpha R \mu} \cdot 2 \cosh \left(\alpha R \sqrt{1-\mu^{2}} \cdot \mu^{*}\right)+ \\
& +\sigma \int_{0}^{R} \frac{\mu}{\mu^{*}} e^{-\alpha R \mu} \cdot 2 \cosh \alpha r \mu^{*} \cdot\left[\frac{\partial}{\partial R} \tilde{J}\left(r ; R, \mu_{0}\right)\right] \mathrm{d} r \cdot H\left(r-R \sqrt{1-\mu^{2}}\right)+ \\
& +\sigma \int_{0}^{R}\left[\frac{R\left(1-\mu^{2}\right)}{r^{2} \mu^{* 2}}-\alpha \mu\right] \cdot \frac{\mu}{\mu^{*}} e^{-\alpha R \mu} \cdot 2 \cosh \alpha r \mu^{*} \times \\
& \times \tilde{J}\left(r ; R, \mu_{0}\right) \mathrm{d} r \cdot H\left(r-R \sqrt{1-\mu^{2}}\right)+ \\
& +\sigma \int_{0}^{R}\left[-\frac{\alpha R\left(1-\mu^{2}\right)}{r \mu^{*}}\right] \cdot \frac{\mu}{\mu^{*}} e^{-\alpha R \mu} \cdot 2 \sinh \alpha r \mu^{*} \times \\
& \times \tilde{J}\left(r ; R, \mu_{0}\right) \mathrm{d} r \cdot H\left(r-R \sqrt{1-\mu^{2}}\right) .
\end{aligned}
$$

Differentiating (3.4.2) again with respect to $\mu$, using (3.5.14) and multiplying by $\left(1-\mu^{2}\right) / R \mu$, we obtain

$$
\begin{aligned}
\frac{1-\mu^{2}}{R \mu} \frac{\partial}{\partial \mu} S & \left(\mu, \mu_{0}, R\right)=\sigma \sqrt{1-\mu^{2}} \cdot \tilde{J}\left(R \sqrt{1-\mu^{2}} ; R, \mu_{0}\right) \frac{\mu}{\mu^{*}} e^{-\alpha R \mu} \times \\
& \times 2 \cosh \left(\alpha R \sqrt{1-\mu^{2}} \cdot \mu^{*}\right)+ \\
& +\sigma \int_{0}^{R}\left[\frac{1-\mu^{2}}{R \mu^{2}}-\frac{R\left(1-\mu^{2}\right)}{r^{2} \mu^{* 2}}-\frac{\alpha\left(1-\mu^{2}\right)}{\mu}\right] \cdot \frac{\mu}{\mu^{*}} e^{-\alpha R \mu} \times \\
& \times 2 \cosh \alpha r \mu^{*} \cdot \tilde{J}\left(r ; R, \mu_{0}\right) \mathrm{d} r \cdot H\left(r-R \sqrt{1-\mu^{2}}\right)+ \\
& +\sigma \int_{0}^{R}\left[\frac{\alpha R\left(1-\mu^{2}\right)}{r \mu^{*}}\right] \cdot \frac{\mu}{\mu^{*}} e^{-\alpha R \mu} \times \\
& \times 2 \sinh \alpha r \mu^{*} \cdot \tilde{J}\left(r ; R, \mu_{0}\right) \mathrm{d} r \cdot H\left(r-R \sqrt{1-\mu^{2}}\right) .
\end{aligned}
$$


From (3.4.2), we obtain also

$$
\begin{aligned}
& -\frac{1-\mu^{2}}{R \mu^{2}} S\left(\mu, \mu_{0}, R\right)+\frac{\alpha}{\mu} S\left(\mu, \mu_{0}, R\right)=\sigma \int_{0}^{R}\left[-\frac{1-\mu^{2}}{R \mu^{2}}+\frac{\alpha}{\mu}\right] \times \\
& \times \frac{\mu}{\mu^{*}} e^{-\alpha R \mu} \cdot 2 \cosh \alpha r \mu^{*} \cdot J\left(r ; R, \mu_{0}\right) \mathrm{d} r \cdot H\left(r-R \sqrt{1-\mu^{2}}\right),
\end{aligned}
$$

Adding (3.5.12), (3.5.15), (3.5.16) and (3.5.17) we get

$$
\begin{aligned}
\frac{\partial}{\partial R} S\left(\mu, \mu_{0}, R\right) & +\frac{1-\mu_{0}^{2}}{R \mu_{0}} \frac{\partial}{\partial \mu_{0}} S\left(\mu, \mu_{0}, R\right)+\frac{1-\mu^{2}}{R \mu} \frac{\partial}{\partial \mu} S\left(\mu, \mu_{0}, R\right)- \\
& -\frac{\mu^{2}+\mu_{0}^{2}}{R \mu^{2} \mu_{0}^{2}} S\left(\mu, \mu_{0}, R\right)+\alpha\left(\frac{1}{\mu}+\frac{1}{\mu_{0}}\right) S\left(\mu, \mu_{0}, R\right)= \\
= & \sigma \tilde{J}\left(R ; R, \mu_{0}\right)\left[1+e^{-2 \alpha R \mu}+\frac{1}{2} \int_{0}^{1} S\left(\mu, \mu_{1}, R\right) \frac{\mathrm{d} \mu_{1}}{\mu_{1}}\right] .
\end{aligned}
$$

But from Equation (3.1.1), using (A.1) of Appendix A,

$$
\begin{aligned}
\tilde{J}\left(R ; R, \mu_{0}\right) & =1+e^{-2 \alpha R \mu_{0}}+\frac{\sigma}{2} \int_{0}^{R} \tilde{J}\left(r ; R, \mu_{0}\right) \mathrm{d} r \int_{0}^{1} e^{-\alpha R \mu_{1}} \cdot 2 \cosh \alpha r \mu_{1}^{*} \cdot \frac{\mathrm{d} \mu_{1}}{\mu_{1}^{*}} \times \\
& \times H\left(\mu_{1}-\sqrt{1-\frac{r^{2}}{R^{2}}}\right)=1+e^{-2 \alpha R \mu_{0}}+\frac{\sigma}{2} \int_{0}^{1} \frac{\mathrm{d} \mu_{1}}{\mu_{1}} \int_{0}^{R} \frac{\mu_{1}}{\mu_{1}^{*}} e^{-\alpha R \mu_{1}} \times \\
& \times 2 \cosh \alpha r \mu_{1}^{*} \cdot \tilde{J}\left(r ; R, \mu_{0}\right) \mathrm{d} r \cdot H\left(r-R \sqrt{1-\mu_{1}^{2}}\right)= \\
& =1+e^{-2 \alpha R \mu_{0}}+\frac{1}{2} \int_{0}^{1} S\left(\mu_{1}, \mu_{0}, R\right) \frac{\mathrm{d} \mu_{1}}{\mu_{1}} .
\end{aligned}
$$

Therefore the integro-different ial equation for $S\left(\mu, \mu_{0}, R\right)$ can be written as

$$
\begin{aligned}
\frac{\partial}{\partial R} S\left(\mu, \mu_{0}, R\right) & +\frac{1-\mu^{2}}{R \mu} \frac{\partial}{\partial \mu} S\left(\mu, \mu_{0}, R\right)+\frac{1-\mu_{0}^{2}}{R \mu_{0}} \frac{\partial}{\partial \mu_{0}} S\left(\mu, \mu_{0}, R\right)-\frac{\mu^{2}+\mu_{0}^{2}}{R \mu^{2} \mu_{0}^{2}} \times \\
& \times S\left(\mu, \mu_{0}, R\right)+\alpha\left(\frac{1}{\mu}+\frac{1}{\mu_{0}}\right) S\left(\mu, \mu_{0}, R\right)=\sigma\left[1+e^{-2 \alpha R \mu_{0}}+\right. \\
& \left.+\frac{1}{2} \int_{0}^{1} S\left(\mu_{1}, \mu_{0}, R\right) \frac{\mathrm{d} \mu_{1}}{\mu_{1}}\right] \cdot\left[1+e^{-2 \alpha R \mu}+\frac{1}{2} \int_{0}^{1} S\left(\mu, \mu_{1}, R\right) \frac{\mathrm{d} \mu_{1}}{\mu_{1}}\right]
\end{aligned}
$$

It is to be solved under the initial condition

$$
S\left(\mu, \mu_{0}, 0\right)=0 \text { for } 0 \leqslant \mu, \mu_{0} \leqslant .
$$


The problem is considered suitable for numerical calculation (cf. Bellman et al. (1966) and Rybicki (1970)). Once $S\left(\mu, \mu_{0}, R\right)$ is known, $I(R, \mu)$ can be calculated from (3.4.3) for the case $B_{1}(r)=0$.

We can, however, establish the integro-differential equation for the source function $J(r)$ for $B_{1}(r) \neq 0$.

3.6. THE INTEGRO-DIFFERENTIAL EQUATION FOR THE SOURCE FUNCTION $J(r)$ FOR $B_{1}(r) \neq 0$

The source function $J(r)$ in this case can be replaced by $J\left(r ; R, \mu_{0}\right)$ to signify its dependence on $R$ and $\mu_{0}$. From (2.10), it is given by

$$
\begin{aligned}
r J(r) \equiv & r J\left(r ; R_{0}, \mu_{0}\right)=\frac{\bar{\omega}}{2} \int_{0}^{R} t J\left(t ; R, \mu_{0}\right) K(r, t) \mathrm{d} t+r B_{1}(r)+ \\
& +\bar{\omega} \frac{F}{4} \frac{R^{2}}{r} \frac{\mu_{0}}{\mu_{0}^{*}} e^{-\alpha R \mu_{0}} \cdot 2 \cosh \alpha r \mu_{0}^{*} \cdot H\left(r-R \sqrt{1-\mu_{0}^{2}}\right) .
\end{aligned}
$$

Then

$$
\begin{aligned}
\frac{\partial}{\partial R}[r J(r ; R & \left.\left., \mu_{0}\right)\right]+\frac{1-\mu_{0}^{2}}{R \mu_{0}} \frac{\partial}{\partial \mu_{0}}\left[r J\left(r ; R, \mu_{0}\right)\right]=\frac{\bar{\omega}}{2} \int_{0}^{R}\left\{\frac{\partial}{\partial R}\left[t J\left(t ; R, \mu_{0}\right)\right]+\right. \\
& \left.+\frac{1-\mu_{0}^{2}}{R \mu_{0}} \frac{\partial}{\partial \mu_{0}}\left[t J\left(t ; R, \mu_{0}\right)\right]\right\} \cdot K(r, t) \mathrm{d} t+\frac{\sigma R^{2}}{2} \frac{T}{r} J\left(R ; R, \mu_{0}\right) \int_{0}^{1} e^{-\alpha R \mu_{1}} \times \\
& \times 2 \cosh \alpha r \mu_{1}^{*} \cdot \frac{\mathrm{d} \mu_{1}}{\mu_{1}^{*}} \cdot H\left(\mu_{1}-\sqrt{1-\frac{r^{2}}{R^{2}}}\right)+\left[\frac{1+\mu_{0}^{2}}{R \mu_{0}^{2}}-\frac{\alpha}{\mu_{0}}\right] \times \\
& \times \frac{\bar{\omega} F}{4} \frac{R^{2}}{r} \frac{\mu_{0}}{\mu_{0}^{*}} e^{-\alpha R \mu_{0}} \cdot 2 \cosh \alpha r \mu_{0}^{*} \cdot H\left(r-R \sqrt{1-\mu_{0}^{2}}\right)
\end{aligned}
$$

In conjunction with the auxiliary Equation (3.1.1), Equation (3.6.2) leads to the following homogeneous integral equation

$$
\Phi_{2}\left(r ; R, \mu_{0}\right)=\frac{\bar{\omega}}{2} \int_{0}^{R} \Phi_{2}\left(t ; R, \mu_{0}\right) K(r, t) \mathrm{d} t,
$$

where

$$
\begin{aligned}
\Phi_{2}\left(r ; R, \mu_{0}\right)=\frac{\partial}{\partial R} & {\left[r J\left(r ; R, \mu_{0}\right)\right]+\frac{1-\mu_{0}^{2}}{R \mu_{0}} \frac{\partial}{\partial \mu_{0}}\left[r J\left(r ; R, \mu_{0}\right)\right]-} \\
& -\frac{\bar{\omega} F}{4} \frac{1+\mu_{0}^{2}}{R \mu_{0}^{2}}\left[r \tilde{J}\left(r ; R, \mu_{0}\right)\right]+\frac{\bar{\omega} F}{4} \frac{\alpha}{\mu_{0}}\left[r \tilde{J}\left(r ; R, \mu_{0}\right)\right]- \\
& -\frac{\sigma}{2} J\left(R ; R, \mu_{0}\right) \int_{0}^{1}\left[r \tilde{J}\left(r ; R, \mu_{1}\right)\right] \frac{\mathrm{d} \mu_{1}}{\mu_{1}}
\end{aligned}
$$


For the non-homogeneous auxiliary equation to have a unique solution

$$
\Phi_{2}\left(r ; R, \mu_{0}\right)=0 .
$$

Therefore,

$$
\begin{aligned}
\frac{\partial}{\partial R}\left[r J\left(r ; R, \mu_{0}\right)\right] & +\frac{1-\mu_{0}^{2}}{R \mu_{0}} \frac{\partial}{\partial \mu_{0}}\left[r J\left(r ; R, \mu_{0}\right)\right]=\frac{\bar{\omega} F}{4} \frac{1+\mu_{0}^{2}}{R \mu_{0}^{2}} \times \\
& \times\left[r \tilde{J}\left(r ; R, \mu_{0}\right)\right]-\frac{\bar{\omega} F}{4} \frac{\alpha}{\mu_{0}}\left[r \tilde{J}\left(r ; R, \mu_{0}\right)\right]+ \\
& +\frac{\sigma}{2} J\left(R ; R, \mu_{0}\right) \int_{0}^{1}\left[r \tilde{J}\left(r ; R, \mu_{1}\right)\right] \frac{\mathrm{d} \mu_{1}}{\mu_{1}} .
\end{aligned}
$$

in which $r \tilde{J}\left(r ; R, \mu_{0}\right)$ is the $N$-solution of the auxiliary equation (cf. (3.1.4)).

\subsection{THE INTEGRO-DIFFERENTIAL EQUATION FOR THE EMERGENT INTENSITY} WHEN $B_{1}(r) \neq 0$

From (3.3.5), replacing $J(r)$ by $J\left(r ; R, \mu_{0}\right)$ to emphasize its dependence on $R$ and $\mu_{0}$, we can write

$$
I\left(R, \mu ; \mu_{0}\right)=\int_{0}^{R} J\left(r ; R, \mu_{0}\right) e^{-\alpha R \mu} \cdot 2 \cosh \alpha r \mu^{*} \cdot \frac{\alpha \mathrm{d} r}{\mu^{*}} \cdot H\left(r-R \sqrt{1-\mu^{2}}\right) .
$$

Multiplying equation (3.6.6) by

$$
\sigma \frac{\mu}{\mu^{*}} e^{-\alpha R \mu} \cdot 2 \cosh \alpha r \mu^{*} \cdot \mathrm{d} r \cdot H\left(r-R \sqrt{1-\mu^{2}}\right),
$$

integrating it over $(0, R)$ and inverting the order of integration on the right-hand side, we get

$$
\begin{aligned}
\sigma \int_{0}^{R} \frac{\mu}{\mu^{*}} e^{-\alpha R \mu} \cdot 2 \cosh \alpha r \mu^{*} \cdot\left[\frac{\partial}{\partial R} J\left(r ; R, \mu_{0}\right)\right] \mathrm{d} r \cdot H\left(r-R \sqrt{1-\mu^{2}}\right)+ \\
+\bar{\omega} \mu \frac{1-\mu_{0}^{2}}{R \mu_{0}} \frac{\partial}{\partial \mu_{0}} I\left(R, \mu ; \mu_{0}\right)=\frac{\bar{\omega} F}{4} \frac{1+\mu_{0}^{2}}{R \mu_{0}^{2}} S\left(\mu, \mu_{0}, R\right)- \\
-\frac{\bar{\omega} F}{4} \frac{\alpha}{\mu_{0}} S\left(\mu, \mu_{0}, R\right)+\frac{\sigma}{2} J\left(R ; R, \mu_{0}\right) \int_{0}^{1} S\left(\mu, \mu_{1}, R\right) \frac{d \mu_{1}}{\mu_{1}},
\end{aligned}
$$

where $I\left(R, \mu ; \mu_{0}\right)$ and $S\left(\mu, \mu_{0}, R\right)$ are given by (3.7.1) and (3.4.2), respectively; and

$$
\begin{gathered}
\frac{\partial}{\partial R} I\left(R, \mu ; \mu_{0}\right)+\frac{1-\mu^{2}}{R \mu} \frac{\partial}{\partial \mu} I\left(R, \mu ; \mu_{0}\right)+\frac{\alpha}{\mu} I\left(R, \mu ; \mu_{0}\right)= \\
=\frac{\alpha}{\mu} J\left(R ; R, \mu_{0}\right)\left[1+e^{-2 \alpha R \mu}\right]+
\end{gathered}
$$




$$
+\alpha \int_{0}^{R}\left[\frac{\partial}{\partial R} J\left(r ; R, \mu_{0}\right)\right] e^{-\alpha R \mu} \cdot 2 \cosh \alpha r \mu^{*} \cdot \frac{\mathrm{d} r}{\mu^{*}} \cdot H\left(r-R \sqrt{1-\mu^{2}}\right) .
$$

Therefore, from (3.7.2) and (3.7.3),

$$
\begin{aligned}
\frac{\partial}{\partial R} I\left(R, \mu ; \mu_{0}\right) & +\frac{1-\mu^{2}}{R \mu} \frac{\partial}{\partial \mu} I\left(R, \mu ; \mu_{0}\right)+\frac{1-\mu_{0}^{2}}{R \mu_{0}} \frac{\partial}{\partial \mu_{0}} I\left(R, \mu ; \mu_{0}\right)+\frac{\alpha}{\mu} I\left(R, \mu ; \mu_{0}\right)= \\
= & \frac{F}{4 \mu} \frac{1+\mu_{0}^{2}}{R \mu_{0}^{2}} S\left(\mu, \mu_{0}, R\right)-\frac{F}{4 \mu} \frac{\alpha}{\mu_{0}} S\left(\mu, \mu_{0}, R\right)+ \\
& +\frac{\alpha}{\mu} J\left(R ; R, \mu_{0}\right)\left[1+e^{-2 \alpha R \mu}+\frac{1}{2} \int_{0}^{1} S\left(\mu, \mu_{1}, R\right) \frac{\mathrm{d} \mu_{1}}{\mu_{1}}\right]
\end{aligned}
$$

But from (3.6.1) (using (A.1) of Appendix A),

$$
J\left(R ; R, \mu_{0}\right)=B_{1}(R)+\frac{\bar{\omega} F}{4}\left(1+e^{-2 \alpha R \mu_{0}}\right)+\frac{\bar{\omega}}{2} \int_{0}^{1} I\left(R, \mu_{1} ; \mu_{0}\right) \mathrm{d} \mu_{1} .
$$

Hence, we get

$$
\begin{aligned}
\frac{\partial}{\partial R} I\left(R, \mu ; \mu_{0}\right) & +\frac{1-\mu^{2}}{R \mu} \frac{\partial}{\partial \mu} I\left(R, \mu ; \mu_{0}\right)+\frac{1-\mu_{0}^{2}}{R \mu_{0}} \frac{\partial}{\partial \mu_{0}} I\left(R, \mu ; \mu_{0}\right)+\frac{\alpha}{\mu} I\left(R, \mu ; \mu_{0}\right)= \\
= & \frac{F}{4 \mu} \frac{1+\mu_{0}^{2}}{R \mu_{0}^{2}} S\left(\mu, \mu_{0}, R\right)-\frac{F}{4 \mu} \frac{\alpha}{\mu_{0}} S\left(\mu, \mu_{0}, R\right)+ \\
+ & \frac{1}{\mu}\left[\alpha B_{1}(R)+\frac{\sigma F}{4}\left(1+e^{-2 \alpha R \mu_{0}}\right)+\frac{\sigma}{2} \int_{0}^{1} I\left(R, \mu_{1} ; \mu_{0}\right) \mathrm{d} \mu_{1}\right] \times \\
\times & {\left[1+e^{-2 \alpha R \mu}+\frac{1}{2} \int_{0}^{1} S\left(\mu, \mu_{1}, R\right) \frac{\mathrm{d} \mu_{1}}{\mu_{1}}\right], }
\end{aligned}
$$

where the scattering function $S\left(\mu, \mu_{0}, R\right)$ is governed by Equation (3.5.20).

Equation (3.7.6) is the requisite equation for solving the emergent intensity. It is to be solved under the initial condition

$$
I\left(0, \mu ; \mu_{0}\right)=0, \quad 0 \leqslant \mu, \mu_{0} \leqslant
$$

by numerical method (cf. Bellman et al., 1966). The above relation can be looked upon as the relation between the emergent intensity $I\left(R, \mu ; \mu_{0}\right)$ (for $\left.B_{1}(r) \neq 0\right)$ and $I(R, \mu)=$ $=(F / 4 \mu) S\left(\mu, \mu_{0}, R\right)$ for $B_{1}(r)=0$. Incidentally it may be mentioned that when $S\left(\mu, \mu_{0}, R\right)$ is known from (3.5.20) and (3.5.21) and $I\left(R, \mu ; \mu_{0}\right)$ from (3.7.6) and (3.7.7), we can evaluate: $J\left(r ; R, \mu_{0}\right)$ from (3.6.6) and (3.7.5). 


\section{Acknowledgements}

It is our pleasure to acknowledge our gratitude to Dr S. J. Wilson and Mr T. K. Leong of the University of Singapore for the benefit of discussions we had during the preparation of this work.

We shall also like to express our sincere gratitude to Prof. S. Ueno for kindly drawing our attention to certain discrepancies in the paper and suggesting ways of improving it.

\section{Appendix A. Evaluation of the Kernel $K(r, R)$}

To show that

$$
K(r, R)=\frac{\alpha R}{r} \int_{0}^{1} e^{-\alpha R \mu_{1}} \cdot 2 \cosh \alpha r \mu_{1}^{*} \cdot \frac{\mathrm{d} \mu_{1}}{\mu_{1}^{*}} \cdot H\left(\mu_{1}-\sqrt{1-\frac{r^{2}}{R^{2}}}\right)
$$

where

$$
0<\mu_{1}, \quad \mu_{1}^{*} \leqslant 1
$$

and

$$
R^{2}\left(1-\mu_{1}^{2}\right)=r^{2}\left(1-\mu_{1}^{* 2}\right)
$$

we have by (2.11) and (2.12)

$$
\begin{aligned}
K(r, R) & =\alpha\left[E_{1}(\alpha(R-r))-E_{1}(\alpha(R+r))\right] \\
& =\alpha\left[\int_{\alpha(R-r)}^{\infty} e^{-u} \frac{\mathrm{d} u}{u}-\int_{\alpha(R+r)}^{\infty} e^{-u} \frac{\mathrm{d} u}{u}\right] \\
& =\alpha\left[\int_{\alpha(R-r)}^{\alpha \sqrt{R^{2}-r^{2}}} e^{-u} \frac{\mathrm{d} u}{u}-\int_{\alpha(R+r)}^{\alpha \sqrt{R^{2}-r^{2}}} e^{-u} \frac{\mathrm{d} u}{u}\right] .
\end{aligned}
$$

Putting $u=\alpha\left(R \mu_{1}-r \mu_{1}^{*}\right)$ into the first integral and $u=\alpha\left(R \mu_{1}+r \mu_{1}^{*}\right)$ into the second integral using (A.2), we obtain from (A.3)

$$
\begin{aligned}
K(r, R) & =\alpha\left[\int_{\sqrt{ }\left(1-\left(r^{2} / R^{2}\right)\right)} e^{-\alpha\left(R \mu_{1}-r \mu_{1}^{*}\right)}\left(\frac{R}{r}\right) \frac{\mathrm{d} \mu_{1}}{\mu_{1}^{*}}+\right. \\
& \left.+\int_{\sqrt{ }\left(1-\left(r^{2} / R^{2}\right)\right)}^{1} e^{-\alpha\left(R \mu_{1}+r \mu_{1}^{*}\right)}\left(\frac{R}{r}\right) \frac{\mathrm{d} \mu_{1}}{\mu_{1}^{*}}\right]= \\
& =\frac{\alpha R}{r} \int_{0}^{1} e^{-\alpha R \mu_{1}} \cdot 2 \cosh \alpha r \mu_{1}^{*} \cdot \frac{\mathrm{d} \mu_{1}}{\mu_{1}^{*}} \cdot H\left(\mu_{1}-\sqrt{1-\frac{r^{2}}{R^{2}}}\right)
\end{aligned}
$$

which proves (A.1).

Furthermore, we note that

$$
H\left(\mu_{1}-\sqrt{1-\frac{r^{2}}{R^{2}}}\right)=H\left(r-R \sqrt{1-\mu_{1}^{2}}\right)
$$




\section{Appendix B - Proof of the Existence of Certain Derivatives and Integrals of $\left[r \tilde{J}\left(r ; R, \mu_{0}\right)\right]$}

The steps (3.5.4), (3.5.5) and (3.5.6) are permissible if we can show that

$$
\frac{\partial}{\partial R}\left[r \tilde{J}\left(r ; R, \mu_{0}\right)\right], \quad \frac{\partial}{\partial \mu_{0}}\left[r J\left(r ; R, \mu_{0}\right)\right]
$$

and

$$
\int_{0}^{1}\left[r \tilde{J}\left(r ; R, \mu_{1}\right)\right] \frac{\mathrm{d} \mu_{1}}{\mu_{1}}
$$

exist.

From (3.1.4) and (3.1.5), since $g\left(t ; R, \mu_{0}\right)$ is non-negative, we have

$$
\begin{gathered}
\frac{\partial}{\partial R}\left[r \tilde{J}\left(r ; R, \mu_{0}\right)\right]=\sum_{\gamma=0}^{\infty} R, \mu_{0} L_{r}^{\gamma}\left\{\frac{\partial}{\partial R} g\left(t ; R, \mu_{0}\right)\right\} \\
\frac{\partial}{\partial \mu_{0}}\left[r \tilde{J}\left(r ; R, \mu_{0}\right)\right]=\sum_{\gamma=0}^{\infty} R, \mu_{0} L_{r}^{\gamma}\left\{\frac{\partial}{\partial \mu_{0}} g\left(t ; R, \mu_{0}\right)\right\}
\end{gathered}
$$

and

$$
\int_{0}^{1}\left[r \tilde{J}\left(r ; R, \mu_{1}\right)\right] \frac{\mathrm{d} \mu_{1}}{\mu_{1}}=\sum_{\gamma=0}^{\infty} R, \mu_{0} \mathrm{~L}_{r}^{\gamma}\left\{\int_{0}^{1} g\left(t ; R, \mu_{1}\right) \frac{\mathrm{d} \mu_{1}}{\mu_{1}}\right\}
$$

where

$$
\begin{aligned}
& \frac{\partial}{\partial R} g\left(t ; R, \mu_{0}\right)=\left[\frac{2}{R}+\frac{R\left(1-\mu_{0}^{2}\right)}{r^{2} \mu_{0}^{* 2}}-\alpha \mu_{0}\right] \times \\
& \times \frac{R^{2}}{r} \frac{\mu_{0}}{\mu_{0}^{*}} e^{-\alpha R \mu_{0}} \cdot 2 \cosh \alpha r \mu_{0}^{*} \cdot H\left(r-R \sqrt{\left.1-\mu_{0}^{2}\right)}+\right. \\
& +\left[-\frac{\alpha R\left(1-\mu_{0}^{2}\right)}{r \mu_{0}^{*}}\right] \frac{R^{2}}{r} \frac{\mu_{0}}{\mu_{0}^{*}} e^{-\alpha R \mu_{0}} \cdot 2 \sinh \alpha r \mu_{0}^{*} \cdot H\left(r-R \sqrt{\left.1-\mu_{0}^{2}\right)}\right. \\
& \frac{\partial}{\partial \mu_{0}} g\left(t ; R, \mu_{0}\right)=\left[\frac{1}{\mu_{0}}-\left(\frac{R}{r}\right)^{2} \frac{\mu_{0}}{\mu_{0}^{*}}-\alpha R\right] \times \\
& \times \frac{R^{2}}{r} \frac{\mu_{0}}{\mu_{0}^{*}} e^{-\alpha R \mu_{0}} \cdot 2 \cosh \alpha r \mu_{0}^{*} \cdot H\left(r-R \sqrt{\left.1-\mu_{0}^{2}\right)}+\right. \\
& +\left[\frac{\alpha R^{2}}{r} \frac{\mu_{0}}{\mu_{0}^{*}}\right] \frac{R^{2}}{r} \frac{\mu_{0}}{\mu_{0}^{*}} e^{-\alpha R \mu_{0}} \cdot 2 \sinh \alpha r \mu_{0}^{*} \cdot H\left(r-R \sqrt{\left.1-H_{0}^{2}\right)}\right.
\end{aligned}
$$

and

$$
\int_{0}^{1} g\left(t ; R, \mu_{1}\right) \frac{\mathrm{d} \mu_{1}}{\mu_{1}}=\frac{R^{2}}{r} \int_{0}^{1} e^{-\alpha R \mu_{1}} \cdot 2 \cosh \alpha r \mu_{1}^{*} \cdot \frac{\mathrm{d} \mu_{1}}{\mu_{1}^{*}} \cdot H\left(\mu_{1}-\sqrt{1-\frac{r^{2}}{R^{2}}}\right) .
$$


By virtue of the Heaviside unit step function defined by (2.7), both $(\partial / \partial R) g\left(t ; R, \mu_{0}\right)$ and $\left(\partial / \partial \mu_{0}\right) g\left(t ; R, \mu_{0}\right)$ are bounded, that is, we can find finite non-negative constants $K_{1}, K_{2}$ such that

and

$$
\left|\frac{\partial}{\partial R} g\left(t ; R, \mu_{0}\right)\right| \leqslant K_{1}
$$

$$
\left|\frac{\partial}{\partial \mu_{0}} g\left(t ; R, \mu_{0}\right)\right| \leqslant K_{2}
$$

for $0 \leqslant t \leqslant R$.

By the theorem proved in Section (3.2), the series (B.1) and (B.2) are uniformly convergent. Therefore $(\partial / \partial R)\left[r \tilde{J}\left(r ; R, \mu_{0}\right)\right]$ and $\left(\partial / \partial \mu_{0}\right)\left[r \tilde{J}\left(r ; R, \mu_{0}\right)\right]$ exist.

To prove the convergence of the series (B.3), we rewrite (B.6) using (A.1) of Appendix A

$$
\begin{aligned}
\int_{0}^{1} g\left(t ; R, \mu_{1}\right) \frac{\mathrm{d} \mu_{1}}{\mu_{1}} & =\frac{R}{\alpha} K(r, R) \\
& =R\left[E_{1}(\alpha(R-r))-E_{1}(\alpha(R+r))\right] \\
& \leqslant R E_{1}(\alpha(R-r)) .
\end{aligned}
$$

The convergence of the series (B.9) will follow if we can show that the series

$$
\sum_{\gamma=0}^{\infty} R, \mu_{0} L_{r}^{\gamma}\left\{E_{1}(\alpha(R-t))\right\}
$$

is convergent.

We have

$$
\begin{aligned}
0 & \leqslant{ }_{R, \mu_{0}} \mathrm{~L}_{r}\left\{E_{1}(\alpha(R-t))\right\}= \\
& =\frac{\bar{\omega}}{2} \int_{0}^{R} E_{1}(\alpha(R-t)) K(r, t) \mathrm{d} t \leqslant \\
& \leqslant \frac{\sigma}{2} \int_{0}^{R} E_{1}(\alpha(R-t)) E_{1}(\alpha|r-t|) \mathrm{d} t .
\end{aligned}
$$

Putting $R-t=x$ into the integral in (B.11), we have

$$
\begin{aligned}
0 & \leqslant{ }_{R, \mu_{0}} \mathrm{~L}_{r}\left\{E_{1}(\alpha(R-t))\right\} \leqslant \\
& \leqslant \frac{\sigma}{2} \int_{0}^{\sigma} E_{1}(\alpha x) E_{1}(\alpha|x-R+t|) \mathrm{d} x \leqslant \\
& \leqslant \frac{\sigma}{2} \int_{0}^{\infty} E_{1}(\alpha x) E_{1}(\alpha|x-R+t|) \mathrm{d} x= \\
& =\bar{\omega} \Lambda_{\alpha(R-t)}\left\{E_{1}(\alpha x)\right\}
\end{aligned}
$$

where $\Lambda_{\tau}$ is Hopf's operator. ( $\tau=\alpha r$ is the optical thickness.) 
According to Busbridge (1961), $\Lambda_{\alpha(R-t)}\left\{E_{1}(\alpha x)\right\}$ is bounded. We write

$$
\Lambda_{\alpha(R-t)}\left\{E_{1}(\alpha x)\right\} \leqslant K
$$

for some finite value of $K$ and $0 \leqslant t \leqslant R$. Therefore (B.12) gives

$$
0 \leqslant{ }_{R, \mu_{0}} \mathrm{~L}_{r}\left\{E_{1}(\alpha(R-t))\right\} \leqslant \bar{\omega} K \leqslant K \quad(0 \leqslant \bar{\omega} \leqslant 1) .
$$

Hence, by the theorem of Section (3.2)

$$
R, \mu_{0} L_{r}^{\gamma}\left\{E_{1}(\alpha(R-t))\right\} \leqslant K \chi^{\gamma-1}
$$

and the convergence of the series (B.10) for $0 \leqslant t<R$ follows.

\section{References}

Bailey, P. B.: 1964, J. Math. Anal. Appl. 8, 144.

Bailey, P. B. and Wing, G. M.: 1964, J. Math. Anal. Appl. 8, 170.

Bellman, R. and Kalaba, R.: 1956, Proc. Nat. Acad. Sci. 42, 629.

Bellman, R., Kalaba, R., and Prestrud, M.: 1963, Invariant Imbedding and Radiative Transfer in Slabs of Finite Thickness American Elsevier Publ. Co., New York.

Bellman, R., Kagiwada, H., Kalaba, R., and Ueno, S.: 1966, RM-5402-PR-RAND External Publ. Bellman, R., Kagiwada, H., Kalaba, R., and Ueno, S.: 1968, J. Math. Phys. 9, 909.

Bellman, R., Kagiwada, H., Kalaba, R., and Ueno, S.: 1969, Icarus 11, 417.

Busbridge, I. W.: 1955, Quart. J. Math., Oxford 2, 6, 218.

Busbridge, I. W.: 1955, Monthly Notices Roy. Astron. Soc. 115, 521; 1956, Monthly Notices Roy. Astron. Soc. 116, 304; 1957, Monthly Notices Roy. Astron. Soc. 117, 516.

Busbridge, I. W.: 1960, The Mathematics of Radiative Transfer, Cambridge University Press, Cambridge.

Busbridge, I. W.: 1961, Astrophys. J. 133, 198.

Cuperman, S., Engelmann, F., and Oxenius, J.: 1963, Phys. Fluids, 6, 108.

Germogenova, T. A.: 1966, Astrophyzika 2, 251.

Hopf, E.: 1934, Mathematical Problems of Radiative Equilibrium (Cambridge Tracts, No. 31).

Kourganoff, V., and Busbridge, I. W.: 1952, Basic Methods of Transfer Problem, Oxford.

Leong, T. K. and Sen, K. K.: 1968, Ann. Astrophys. 31, 467.

Leong, T. K. and Sen, K. K.: 1969, Publ. Astron. Soc. Japan 21, 167.

Leong, T. K. and Sen, K. K.: 1970, Publ. Astron. Soc. Japan 22, 57.

Rybicki, G. B.: 1970, J. Comp. Phys. 6, 131.

Sobolev, V. V.: 1963, A Treatise of Radiative Transfer, Van Nostrand, New York.

Tsujita, J.: 1967, Publ. Astron. Soc. Japan 19, 468.

Ueno, S.: 1957, Astrophys. J. 126, 413.

Ueno, S.: 1958, J. Math. Mech. 7, 629.

Ueno, S.: 1959, Ann. Astron. 22, 468 and 484.

Ueno, S.: 1960, Astrophys. J. 132, 729.

Ueno, S., Kagiwada, H., and Kalaba, R.: 1969: The Rand Corporation Research Memorandum, RM-6061-PR.

Uesugi, A., and Tsujita, J.: 1969, Publ. Astron. Soc. Japan 21, 370. 